\title{
Uptake, Translocation, and Metabolism of 8:2 Fluorotelomer Alcohol in Soybean (Glycine max L. Merrill)
}

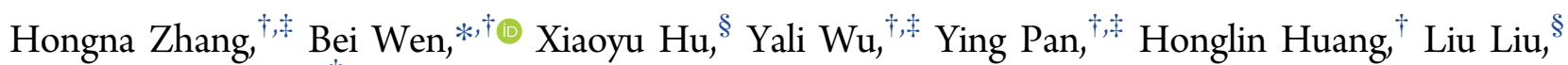 \\ and Shuzhen Zhang
}
${ }^{\dagger}$ State Key Laboratory of Environmental Chemistry and Ecotoxicology, Research Center for Eco-Environmental Sciences, Chinese Academy of Sciences, Beijing 100085, China
${ }^{\ddagger}$ University of Chinese Academy of Sciences, Beijing 100049, China
${ }^{\S}$ Beijing Center for Disease Prevention and Control, Beijing 100020, China

\section{Supporting Information}

ABSTRACT: Biotransformation of fluorotelomer alcohols (FTOHs) is widely considered as an additional source of perfluorocarboxylic acids (PFCAs) in environmental biota. Compared with the extensive studies conducted in animals and microbes, biotransformation pathways of FTOHs in plants are still unclear. In this study, a hydroponic experiment was conducted to investigate the uptake, translocation and metabolism of 8:2 FTOH in soybean (Glycine max L. Merrill) over $144 \mathrm{~h}$. 8:2 FTOH and its metabolites were found in all parts of soybean plants. At the end of the exposure, 7:3 FTCA $\left[\mathrm{F}\left(\mathrm{CF}_{2}\right)_{7} \mathrm{CH}_{2} \mathrm{CH}_{2} \mathrm{COOH}\right]$ was the primary metabolite in roots and stems, while PFOA $\left[\mathrm{F}\left(\mathrm{CF}_{2}\right)_{7} \mathrm{COOH}\right]$ was predominant in leaves. PFOA and 7:3 FTCA in the soybean-solution system accounted for 6.01 and $5.57 \mathrm{~mol} \%$ of the initially applied 8:2 FTOH, respectively. Low levels of PFHpA $\left[\mathrm{F}\left(\mathrm{CF}_{2}\right)_{6} \mathrm{COOH}\right]$ and PFHxA $\left[\mathrm{F}\left(\mathrm{CF}_{2}\right)_{5} \mathrm{COOH}\right]$ in solutions and soybean roots resulted from microbial metabolism and plant root uptake. Glutathione-conjugated metabolites in soybean tissues were also identified. The activities of alcohol dehydrogenase, aldehyde dehydrogenase, and glutathione S-transferase in soybean roots increased during the exposure, suggesting their roles in 8:2 FTOH metabolism in soybean. This study provides important information for a better understanding of the uptake and metabolism of FTOHs and fluorotelomer-based compounds in plants.

\section{INTRODUCTION}

Perfluorocarboxylic acids (PFCAs) are anthropogenic chemicals with a wide range of industrial and consumer applications, and have been found globally in various environmental media and biota. ${ }^{1}$ The toxicity, bioaccumulation and persistence of PFCAs have spurred efforts to determine their sources. In addition to direct emissions from product manufacturing, use and disposal, the environmental occurrence of PFCAs can also arise from indirect transformation of polyfluoroalkyl precursor chemicals. ${ }^{2}$ A number of highly fluorinated chemicals have been subjected to abiotic and biotic degradation studies, and fluorotelomer alcohols (FTOHs) are widely recognized as precursors of PFCAs. $^{3-5}$

FTOHs are raw materials used to produce fluorotelomer intermediates, surfactants, and polymeric materials, and have been found as unreacted and unbound residuals in fluorotelomer-based manufactured products. ${ }^{2,6}$ The breakage of the ester and urethane linkages on the carbon backbones of fluorotelomer-based substances is suspected to release FTOHs into the environment over time. ${ }^{7,8}$ To better comprehend the environmental fate of these fluorotelomer-based substances, it is essential to understand FTOH degradation rates and pathways in different environmental and biotic compartments. In the homologous series of FTOHs, 8:2 FTOH has historically been produced in the largest volume. Degradation of $8: 2 \mathrm{FTOH}$ by oxidation in the atmosphere, ${ }^{9,10}$ photolysis in aqueous environments, ${ }^{11}$ aerobic and anaerobic microbial biodegradation in terrestrial environments, ${ }^{12-14}$ and in vivo and in vitro metabolism in animals ${ }^{15-18}$ and in humans ${ }^{19,20}$ has been reported. The 8:2 FTOH degradation in different matrixes shares some common steps, proceeding via saturated and unsaturated fluorotelomer carboxylic acid intermediates, which are finally oxidized to PFCAs as the terminal products. Perfluorooctanoic acid (PFOA) has been found as a terminal degradation product in almost all of the 8:2 FTOH degradation studies, with the largest molar yield being $40 \%$ in an aerobic soil incubated for up to 7 months. ${ }^{13}$ In addition to PFOA,

Received: July 25, 2016

Revised: November 6, 2016

Accepted: November 18, 2016

Published: November 18, 2016 
degradation of 8:2 FTOH could also yield lower levels of other PFCAs, such as perfluorohexanoic acid (PFHxA), ${ }^{12,13}$ perfluoroheptanoic acid (PFHpA), ${ }^{12}$ and perfluorononanoic acid (PFNA). ${ }^{16,21}$ Besides phase I (oxidation) metabolism, phase II metabolic pathways of 8:2 FTOH through the conjugation of the parent compound and metabolites with glutathione-S-transferase (GST), UDP-glucuronosyltransferase (UGT) and sulfotransferase (ST) in rat and rainbow trout have also been reported. ${ }^{15,17}$

Sewage sludge generated in wastewater treatment plants is widely considered to be an important sink of per- and polyfluoroalkyl substances. ${ }^{22,23}$ Land application of treated sewage sludge (biosolids) may present an exposure route for these contaminants into soils. ${ }^{24-26}$ Yoo et al. ${ }^{26}$ reported a total concentration of FTOHs as high as $169 \mathrm{ng} \cdot \mathrm{g}^{-1}$ dry weight $(\mathrm{dw})$ in sludge-applied soils, with 8:2 FTOH ranging from 5 to $73 \mathrm{ng}$. $\mathrm{g}^{-1} \mathrm{dw}$. In our previous study, FTOHs and their degradation products were found in soils and crops collected from a biosolidsamended agricultural field, suggesting the transfer and degradation potential of FTOHs in soil-plant systems. ${ }^{27}$ The degradation products of FTOHs in plants are the integrated effects of plant degradation and plant uptake from soils. Rankin et $\mathrm{al}^{28}$ reported the biodegradation products of fluorotelomerbased acrylate polymer (FTACP) in a soil-plant microcosm system; however, neither the FTACP nor 8:2 FTOH level was determined in plants. In previous studies, aerobic and anaerobic biodegradation of 8:2 FTOH in soils have been studied extensively, ${ }^{4}$ however, the uptake and metabolic pathways of 8:2 FTOH in plants are still not known.

Therefore, the objective of this study was to explore the uptake, translocation and metabolism of 8:2 FTOH in plants. Soybean was chosen as the model plant because it is one of the most important economic crops worldwide. Hydroponic experiments were conducted in order to minimize the influence of soil microorganisms on the degradation of FTOHs, although the effects of root-associated microbes cannot be entirely excluded. Time-dependent concentrations of 8:2 FTOH and its phase I degradation products were determined in exposure solutions and different parts of soybean plants. Phase II metabolites in soybean tissues were identified. The activities of enzymes, including alcohol dehydrogenase $(\mathrm{ADH})$, aldehyde dehydrogenase $(\mathrm{ALDH}), \mathrm{GST}$, and cytochrome P450 (CYP450), in soybean roots over different exposure times were also analyzed in order to identify the enzymes involved in plant degradation of $8: 2 \mathrm{FTOH}$.

\section{MATERIALS AND METHODS}

Chemicals and Reagents. Chemical names, acronyms and molecular structures of the poly- and perfluorinated substances described in this paper are shown in Table S1 of the Supporting Information (SI). Standards of 8:2 FTOH were purchased from Matrix Scientific (Columbia, SC). PFBA, PFPeA, PFHxA, and PFHpA were from J\&K Scientific Ltd. (Beijing, China). PFOA was from Strem Chemicals Inc. (Newburyport, MA). PFNA was from Alfa Aesar (Ward Hill, MA). 7:3 FTCA were from Apollo Scientific Ltd. (Bredbury, UK). 8:2 FTCA, 8:2 FTUCA, 7:2 sFTOH, and stable isotope-labeled standards (M8:2 FTOH, M8:2 FTCA, M8:2 FTUCA, MPFBA, MPFHxA, MPFOA, MPFNA) were from Wellington Laboratories Inc. (Guelph, Ontario, Canada). Purities of all the analytical standards were $\geq 97 \%$. Solvents, including methanol, acetonitrile, and ethyl acetate were of HPLC grade (Fisher Chemical, Fairlawn, NJ). All the other chemicals used were of analytical grade or better. Purified water obtained by a Milli-Q Synthesis water purification system (Millipore, Bedford, MA, USA) and deionized water were used in the experiments.

Plant Culture and Exposure Experiments. Soybean (Glycine $\max$ L. Merrill) seeds were obtained from the Chinese Academy of Agricultural Sciences (Beijing, China). Seeds were first surface-sterilized with $10 \% \mathrm{H}_{2} \mathrm{O}_{2}$ solution for $15 \mathrm{~min}$, thoroughly rinsed and soaked overnight in autoclaved deionized water at room temperature, and then germinated on moist filter paper for 5 days at $27{ }^{\circ} \mathrm{C}$ in the dark. After germination, the seedlings were transferred to containers containing sterile nutrient solution (1/4-strength modified Hoagland's solution, ${ }^{29}$ SI Table S2) for cultivation.

After 8 days, three uniformly germinated seedlings were transferred to autoclaved $150 \mathrm{~mL}$ brown glass-stoppered flasks, which were used as the exposure reactors. Each reactor was filled with $120 \mathrm{~mL}$ of sterile nutrient solution and $25.8 \mathrm{nmol}$ of $8: 2$ FTOH (dissolved in $60 \mu \mathrm{L}$ of methanol), giving an initial exposure concentration of $215 \mathrm{nmol} \cdot \mathrm{L}^{-1}$. The soybean seedlings were transferred to the reactor immediately after 8:2 FTOH spiking. A sealed test system with a rubber plug was used to prevent the volatilization of 8:2 FTOH. The detailed scheme of the exposure reactor is described in SI Figure S1. The reactor was wrapped with light-proof paper to eliminate the photolysis of FTOHs. Soybean plants wrapped with thin sponge on the stem grew through a predrilled hole in the rubber plug. Compensated solution was injected into the reactor through a tube, which was wrapped with light-proof paper and sealed with Parafilm during the experiments.

The experiments were conducted in a laminar flow hood and all experimental solutions and materials were autoclaved before use. The reactors were then put into a growth chamber with 14 $\mathrm{h} / 10 \mathrm{~h}$ day/night photoperiod and light intensity of $250 \mu \mathrm{mol}$. $\mathrm{m}^{-2} \cdot \mathrm{s}^{-1}$, and day/night temperature regime of $25^{\circ} \mathrm{C} / 22^{\circ} \mathrm{C}$. All reactors were positioned randomly and rerandomized every day. Approximately $30 \mathrm{~mL} \cdot \mathrm{d}^{-1}$ of sterile nutrient solution was injected into reactors with plants to compensate for the transpiration losses, according to the weight of the reactors (gravimetric determination). Soybean seedlings were sampled at intervals of $12,24,48,72,96$, and $144 \mathrm{~h}$. The experimental systems in which soybean seedlings were incubated in 8:2 FTOH-spiked culture solution were defined as whole soybean. Unplanted controls (with 8:2 FTOH but without seedlings) and blank controls (with seedlings but without 8:2 FTOH) were set up simultaneously. Concentrations of 8:2 FTOH and its metabolites in soybean tissues and solutions, and activities of $\mathrm{ADH}, \mathrm{ALDH}, \mathrm{GST}$, and CYP450 in soybean roots were analyzed. The distribution of 8:2 FTOH in the reactors of unplanted controls was tested after the addition of 8:2 FTOH for $144 \mathrm{~h}$.

Ethanol-washed soybean controls were set up to evaluate the role of microbes in 8:2 FTOH metabolite patterns in the solution and soybean plants. The seedling roots were washed with $70 \%$ ethanol solution for $30 \mathrm{~s}$ and then rinsed with autoclaved deionized water prior to exposure to 8:2 FTOH. After exposure for $144 \mathrm{~h}$, the seedlings were harvested, and 8:2 FTOH and its metabolites in soybean tissues and solutions were determined.

Root exudate controls were conducted to evaluate the effects of plant exudates on microbial degradation of 8:2 FTOH in solutions. Three soybean seedlings were planted in reactors containing $120 \mathrm{~mL}$ sterile nutrient solution without 8:2 FTOH. The seedlings were taken out after 6 days, leaving plant root exudates in the solution. The solutions were replenished to 120 $\mathrm{mL}$ with sterile nutrient solution. 8:2 FTOH was spiked into the 

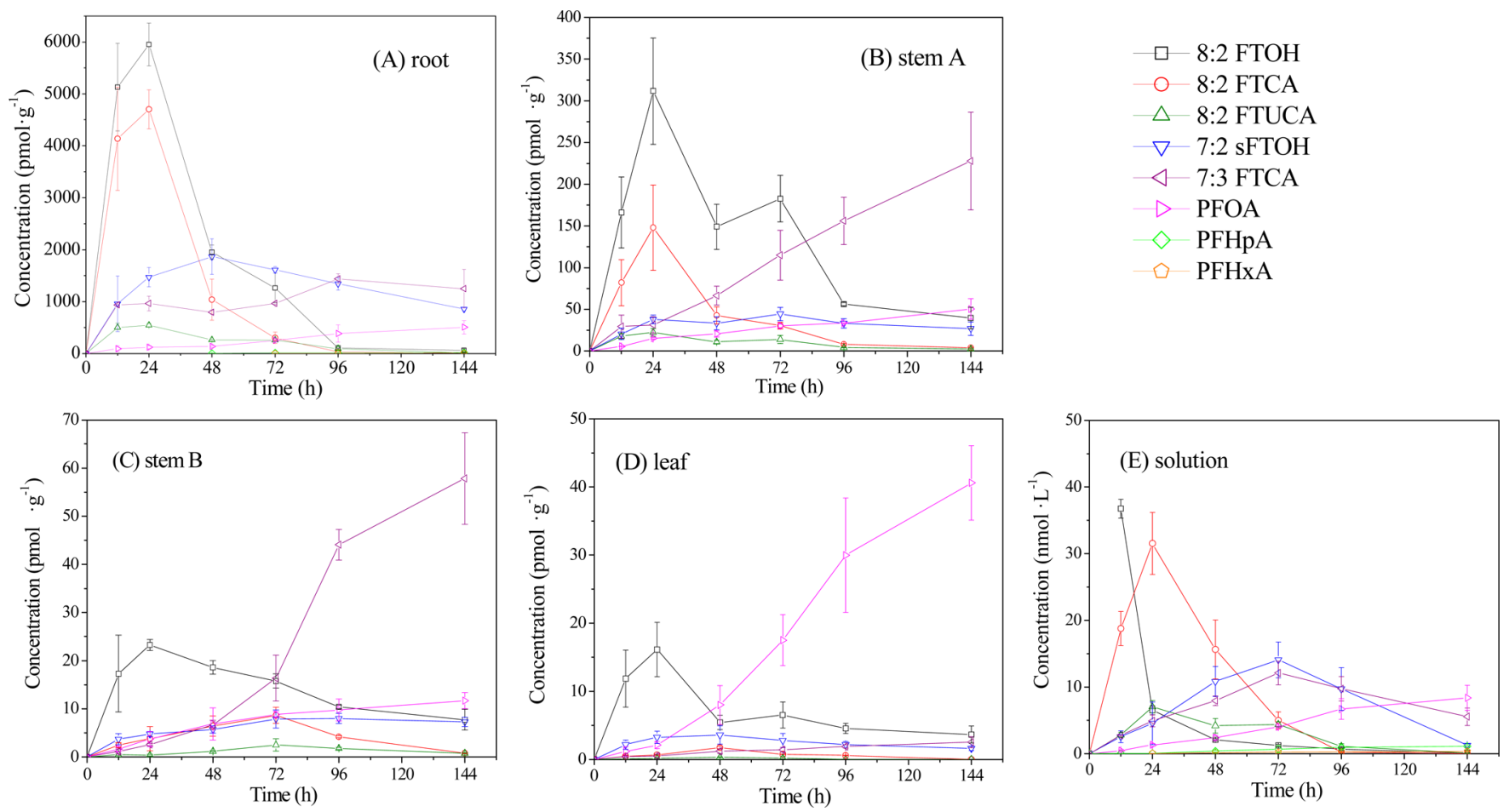

Figure 1. Time-dependent concentrations of 8:2 FTOH and quantified metabolites in different parts of whole soybean and in culture solutions. The curve of 8:2 FTOH in solutions started at $12 \mathrm{~h}$ (the initial concentration was $167 \mathrm{nmol} \cdot \mathrm{L}^{-1}$ ).

reactors with the final concentration of $215 \mathrm{nmol} \cdot \mathrm{L}^{-1}$. The reactors were put into the growth chamber and sampled at intervals of $12,24,48,72,96$, and $144 \mathrm{~h}$. Two treatments were conducted simultaneously. $\mathrm{NaN}_{3}$ controls were set up by adding $\mathrm{NaN}_{3}(0.02 \%, w / w)$ to the solution containing plant exudates before 8:2 FTOH spiking. Autoclaved controls were conducted by putting reactors containing plant exudates into an autoclave sterilizer at $121{ }^{\circ} \mathrm{C}$ for $20 \mathrm{~min}$ before 8:2 FTOH spiking. Each treatment was sampled at $144 \mathrm{~h}$. 8:2 FTOH and its metabolites in solutions were determined. All experiments were performed in triplicate.

Sample Preparation and Enzyme Assays. Root samples were carefully rinsed with sterile water, and rinses were collected and combined with the exposure solutions for the analysis of 8:2 FTOH and its metabolites. Approximately $0.5 \mathrm{~g}$ of fresh roots (except for alcohol-washed plant controls) were homogenized on ice with $4 \mathrm{~mL}$ of extraction buffer $(\mathrm{pH} 7.8)$ containing $0.05 \mathrm{M}$ $\mathrm{NaH}_{2} \mathrm{PO}_{4}+\mathrm{Na}_{2} \mathrm{HPO}_{4}, 2$ mM EDTA, $1 \%$ (w/v) polyvinylpyrrolidone and $2 \mathrm{mM}$ dithiothreitol. After centrifugation at 12000 $\mathrm{rpm}$ at $4{ }^{\circ} \mathrm{C}$ for $15 \mathrm{~min}$, the supernatant was collected for further analysis. The protein level was measured by the Bradford method $^{30}$ using bovine serum albumin as the standard. Enzyme activities of $\mathrm{ADH}, \mathrm{ALDH}, \mathrm{GST}$, and CYP450 were determined spectrophotometrically at $25{ }^{\circ} \mathrm{C}$. Detailed information on the enzyme assays is available in the SI. All the enzyme activities are expressed as $\mathrm{U} \cdot \mathrm{mg}^{-1}$ protein.

Other plant tissue samples (roots, stems and leaves) and solution samples were used for quantitative determination of $8: 2$ FTOH and its degradation products. The tissues were freezedried at $-50{ }^{\circ} \mathrm{C}$ for $48 \mathrm{~h}$ in a lyophilizer (FD-1, Boyikang Instrument Ltd., Beijing, China), weighed and stored at $-20^{\circ} \mathrm{C}$ for analysis. The subsequent calculations were based on dry weight. The solution samples were extracted immediately after sampling. The analyte extraction and cleanup procedures were based on our previous paper ${ }^{27}$ with some modifications.
Surrogate standards (M8:2 FTOH, M8:2 FTCA, M8:2 FTUCA, MPFBA, MPFHxA, MPFOA, MPFNA, 5 ng of each) were added to the samples prior to extraction. Details of sample extraction and cleanup of 8:2 FTOH and its metabolites in plant and solution samples are provided in the SI.

The distribution of 8:2 FTOH in unplanted controls sampled at $144 \mathrm{~h}$ was determined. Extractions were carried out for the reactor and the rubber plug after culture solution decanting. Further details of the extractions can be found in the SI.

Instrumental Analysis. The instrumental analysis was performed by a Waters ultraperformance liquid chromatography system coupled to a Xevo TQS tandem mass spectrometer (UPLC-MS/MS) with an electrospray ionization interface. FTOHs (8:2 FTOH and 7:2 sFTOH) and acid degradation products in purified sample extracts were quantified using an ACQUITY UPLC BEH Shield RP18 column (1.7 $\mu$ m particles, $150 \mathrm{~mm} \times 2.1 \mathrm{~mm}$, Waters $)$ and BEH C18 column $(1.7 \mu \mathrm{m}$ particles, $50 \mathrm{~mm} \times 2.1 \mathrm{~mm}$, Waters), respectively. Electrospray ionization was operated in negative mode, and multiple reaction monitoring (MRM) was used for all analysis. Details of the UPLC gradient, mass spectrometer operational parameters and quantification ion transitions for each of the analytes have been reported in our previous paper ${ }^{27}$ and are summarized in Tables S3 and S4 of the SI. Glutathione (GSH)-conjugated metabolites were detected in soybean tissue extracts using an ACQUITY UPLC BEH Shield RP18 column $(1.7 \mu$ m particles, $150 \mathrm{~mm} \times$ $2.1 \mathrm{~mm}$, Waters) with $2 \mathrm{mM}$ ammonium acetate and methanol as mobile phases. The standards were not available and thus conjugated metabolites were quantified using arbitrary units. Detailed information on the instrumental method is shown in SI Table S5. The MassLynx Software v.4.1 (Waters) was used for instrument control, data acquisition, and processing.

Quality Control/Quality Assurance. Quality control was performed by regular analyses of procedural blanks, blind duplicate samples, and random injection of solvent blanks and 
standards. The accuracy of the determination was assessed by testing the recoveries of isotope-labeled surrogate standards from the spiked blank matrixes in comparison to matrix-matched standards. Compounds with no isotope-labeled standard were corrected with the closest available surrogate standard, as shown in SI Table S6. Recoveries of the seven different isotope-labeled surrogate standards ranged from $68.2 \%$ to $107.8 \%$ for plant tissue samples (roots, stems, and leaves), and from $71.7 \%$ to $111.6 \%$ for solution samples. Compound loss and cross contamination during freeze-drying was negligible (SI Table S7). The limits of detection (LODs) and quantification (LOQs) were defined as the concentrations corresponding to the mean peak area plus three standard deviations $(x+3 \times \sigma)$ or plus 10 standard deviations $(x+10 \times \sigma)$ of extracts from the blanks. Details of the individual LODs and LOQs are listed in SI Table S8.

\section{RESULTS AND DISCUSSION}

Experimental System. In this study, the nominal concentration of 8:2 FTOH in the spiked culture solution was $215 \mathrm{nmol} \cdot \mathrm{L}^{-1}$, lower than its reported aqueous solubility of 295$599 \mathrm{nmol} \cdot \mathrm{L}^{-1}$ at $22-25{ }^{\circ} \mathrm{C} .^{31-33}$ The initial concentration determined in spiked culture solution was $167 \pm 15 \mathrm{nmol} \cdot \mathrm{L}^{-1}$, which was $77.7 \%$ of the nominal concentration. The value determined was used for mass balance calculations. After $144 \mathrm{~h}$, the concentration of $8: 2 \mathrm{FTOH}$ in the solution of unplanted controls was $38.5 \pm 5.2 \mathrm{nmol} \cdot \mathrm{L}^{-1}$. The amounts of $8: 2 \mathrm{FTOH}$ absorbed to the reactor and the rubber plug were $0.27 \pm 0.10 \mathrm{~mol}$ $\%$ and $14.6 \pm 2.72 \mathrm{~mol} \%$ of initially applied 8:2 FTOH, respectively. Total recovery of 8:2 FTOH in unplanted controls was $38 \mathrm{~mol} \%$ (SI Table S9). No degradation product was detected. 8:2 FTOH in aqueous solution can migrate rapidly to the water-air interface and partition quantitatively into air due to its relatively high water-air partitioning coefficient. ${ }^{34}$ The low recovery of unplanted controls suggested the evaporation of 8:2 FTOH within the reactor. The finding of 8:2 FTOH adsorbed to the rubber plug also indicated the evaporation. This result was consistent with those obtained by Wang et al. ${ }^{35}$ and $\mathrm{Nabb}$ et al., ${ }^{16}$ who found that 8:2 FTOH evaporated from the aqueous fraction and migrated into the headspace in their experimental systems. No significant difference was found in soybean dry weights between those of the whole soybean systems and the blank controls at each time point (two-tailed unpaired Student's $t$ test, $P>0.05$, SI Figure S2).

Concentrations of 8:2 FTOH and Its Degradation Products in Soybean Plants. During the exposure time, the levels of 8:2 FTOH and metabolites in plant tissues of the blank controls were under their detection limits, suggesting negligible 8:2 FTOH aerial absorption from air outside of the reactor. Time-dependent concentrations of 8:2 FTOH in roots, stems A and $\mathrm{B}$, and leaves of whole soybean are depicted in Figure 1A-D. The distribution of 8:2 FTOH followed the descending order roots $>$ stem $\mathrm{A}>$ stem $\mathrm{B}>$ leaves. The accumulations of $8: 2$ FTOH in stem $\mathrm{B}$ and leaves indicated its acropetal translocation in soybean. 8:2 FTOH concentrations in stem A were 4.0-18.9 times higher than those in stem $B$, which may be the integrated results of root uptake and acropetal translocation, and absorption on stem A from the culture solution and from the gas phase in the reactor. The concentrations of 8:2 FTOH reached peak levels at $24 \mathrm{~h}$ in all tissues and decreased thereafter.

Five transformation products, 8:2 FTCA, 8:2 FTUCA, 7:3 FTCA, 7:2 sFTOH, and PFOA, were observed in all parts of soybean plants at the first sampling time $(12 \mathrm{~h})$, but PFHpA and PFHxA were found at low levels in soybean roots only after exposure for 48 and $72 \mathrm{~h}$, respectively (Figure 1A-D, SI Table S10). Metabolite concentrations in soybean tissues followed the order: roots > stem A > stem B > leaves. The levels of 8:2 FTCA, 8:2 FTUCA, and 7:2 sFTOH followed the same pattern, first increasing at different rates and then decreasing. 8:2 FTCA and 8:2 FTUCA generally reached peak values faster than 7:2 sFTOH, indicating that $7: 2$ sFTOH could be taken as a downstream degradation product. 7:3 FTCA and PFOA in plant tissues basically kept increasing during the exposure time. At the time of last sampling, 7:3 FTCA was the predominant metabolite in soybean roots $\left(1247.7 \pm 368.6 \mathrm{pmol} \cdot \mathrm{g}^{-1}\right)$, stem A $(227.8 \pm$ $\left.58.6 \mathrm{pmol} \cdot \mathrm{g}^{-1}\right)$, and stem B $\left(57.8 \pm 9.5 \mathrm{pmol} \cdot \mathrm{g}^{-1}\right)$, while PFOA was predominant in leaves $\left(40.6 \pm 5.5 \mathrm{pmol} \cdot \mathrm{g}^{-1}\right)$.

Metabolites in soybean were the integrated results of plant degradation and plant root absorption from culture solutions. If only the process of uptake occurs, the parent and metabolite concentrations in plants should finally approach equilibrium. ${ }^{36}$ However, results of this study showed that levels of $8: 2 \mathrm{FTOH}$, 8:2 FTCA, 8:2 FTUCA, and 7:2 sFTOH in different soybean tissues clearly decreased after reaching a peak level. These patterns indicated that $8: 2 \mathrm{FTOH}$ could be degraded to intermediates and further to stable degradation products in soybean. The increasing trends of PFOA and 7:3 FTCA suggested that they are stable metabolites in soybean tissues.

Concentrations of 8:2 FTOH and Its Degradation Products in Solutions. Time-dependent concentrations of 8:2 FTOH and metabolites in culture solutions are displayed in Figure 1E. The 8:2 FTOH level decreased rapidly to less than 40 $\mathrm{nmol} \cdot \mathrm{L}^{-1}$ within $12 \mathrm{~h}$, and kept declining to $0.11 \mathrm{nmol} \cdot \mathrm{L}^{-1}$ at 144 h. Degradation products 8:2 FTCA, 8:2 FTUCA, 7:3 FTCA, 7:2 sFTOH, PFOA, and PFHpA were observed at the time of first sampling, whereas PFHxA was observed at the time of second sampling. The variation trends for concentrations of 8:2 FTCA, 8:2 FTUCA, and 7:2 sFTOH were similar to those in soybean, increasing initially and then decreasing. Unlike those in soybean, 7:3 FTCA levels in solutions did not continue to increase during exposure, but showed a maximum at $72 \mathrm{~h}$ followed by a decrease. Concentrations of PFOA, PFHpA, and PFHxA increased continuously over the exposure time. At the last sampling time, the sequence of metabolite levels in solutions was PFOA, 7:3 FTCA, 7:2 sFTOH, PFHpA, PFHxA, 8:2 FTUCA, and 8:2 FTCA, in descending order.

Metabolites in culture solution most likely originated from degradation of 8:2 FTOH by microbes in solutions and root releases after plant degradation, similar to what was observed for trifluralin and lindane while they were taken up by ryegrass from hydroponic solution. ${ }^{37}$ Although the reactors were closed and sterile, root-associated microbes such as rhizobia and rhizobacteria $^{38}$ could be inoculated in the solution as a result of plant cultivation. Compared with those of whole soybean systems, more 8:2 FTOH, 8:2 FTCA, 8:2 FTUCA, and 7:2 sFTOH, while less 7:3 FTCA, PFOA, PFHpA, and PFHxA were found in the solutions and plant roots of ethanol-washed soybean controls. However, 8:2 FTOH and its metabolite levels in stems and leaves of ethanol-washed soybean were much higher than those of whole soybean (SI Figure S3). This could be explained by the fact that $70 \%$ ethanol killed a significant fraction of root-associated microbes and partially damaged the roots at the beginning of the exposure, thus decreasing the degradation rates of 8:2 FTOH in both solutions and plant roots. As a result, more 8:2 FTOH was accumulated in plant roots, translocated from roots to the above parts of the plant, and degraded in stems and leaves. 
Table 1. Concentrations of 8:2 FTOH and Its Metabolites in Solutions Containing Plant Exudates after 8:2 FTOH Exposure for $144 \mathrm{~h}$

\begin{tabular}{|c|c|c|c|c|c|c|c|c|}
\hline control type & $\begin{array}{l}8: 2 \mathrm{FTOH} \\
\left(\mathrm{nmol} \cdot \mathrm{L}^{-1}\right)\end{array}$ & $\begin{array}{l}8: 2 \text { FTCA } \\
\left(\mathrm{nmol} \cdot \mathrm{L}^{-1}\right)\end{array}$ & $\begin{array}{c}8: 2 \text { FTUCA } \\
\left(\mathrm{nmol} \cdot \mathrm{L}^{-1}\right)\end{array}$ & $\begin{array}{l}\text { 7:2 sFTOH } \\
\left(\mathrm{nmol} \cdot \mathrm{L}^{-1}\right)\end{array}$ & $\begin{array}{l}\text { 7:3 FTCA } \\
\left(\mathrm{nmol} \cdot \mathrm{L}^{-1}\right)\end{array}$ & $\begin{array}{c}\text { PFOA } \\
\left(\mathrm{nmol} \cdot \mathrm{L}^{-1}\right)\end{array}$ & $\begin{array}{c}\text { PFHpA } \\
\left(\mathrm{nmol} \cdot \mathrm{L}^{-1}\right)\end{array}$ & $\begin{array}{c}\text { PFHxA } \\
\left(\mathrm{nmol} \cdot \mathrm{L}^{-1}\right)\end{array}$ \\
\hline root exudate controls & $1.18 \pm 0.53^{a}$ & $0.40 \pm 0.09$ & $14.8 \pm 2.9$ & $23.6 \pm 2.5$ & $24.0 \pm 2.8$ & $1.75 \pm 0.45$ & $4.24 \pm 1.22$ & $0.41 \pm 0.02$ \\
\hline $\mathrm{NaN}_{3}$ controls & $32.8 \pm 6.7$ & $8.65 \pm 0.82$ & $3.59 \pm 0.28$ & $0.95 \pm 0.31$ & $0.54 \pm 0.19$ & $0.07 \pm 0.02$ & $0.14 \pm 0.03$ & $<$ LOD \\
\hline autoclaved controls & $40.1 \pm 5.2$ & $<$ LOD & $<$ LOD & $<$ LOD & $<$ LOD & $<$ LOD & $<\mathrm{LOD}$ & $<\mathrm{LOD}$ \\
\hline
\end{tabular}
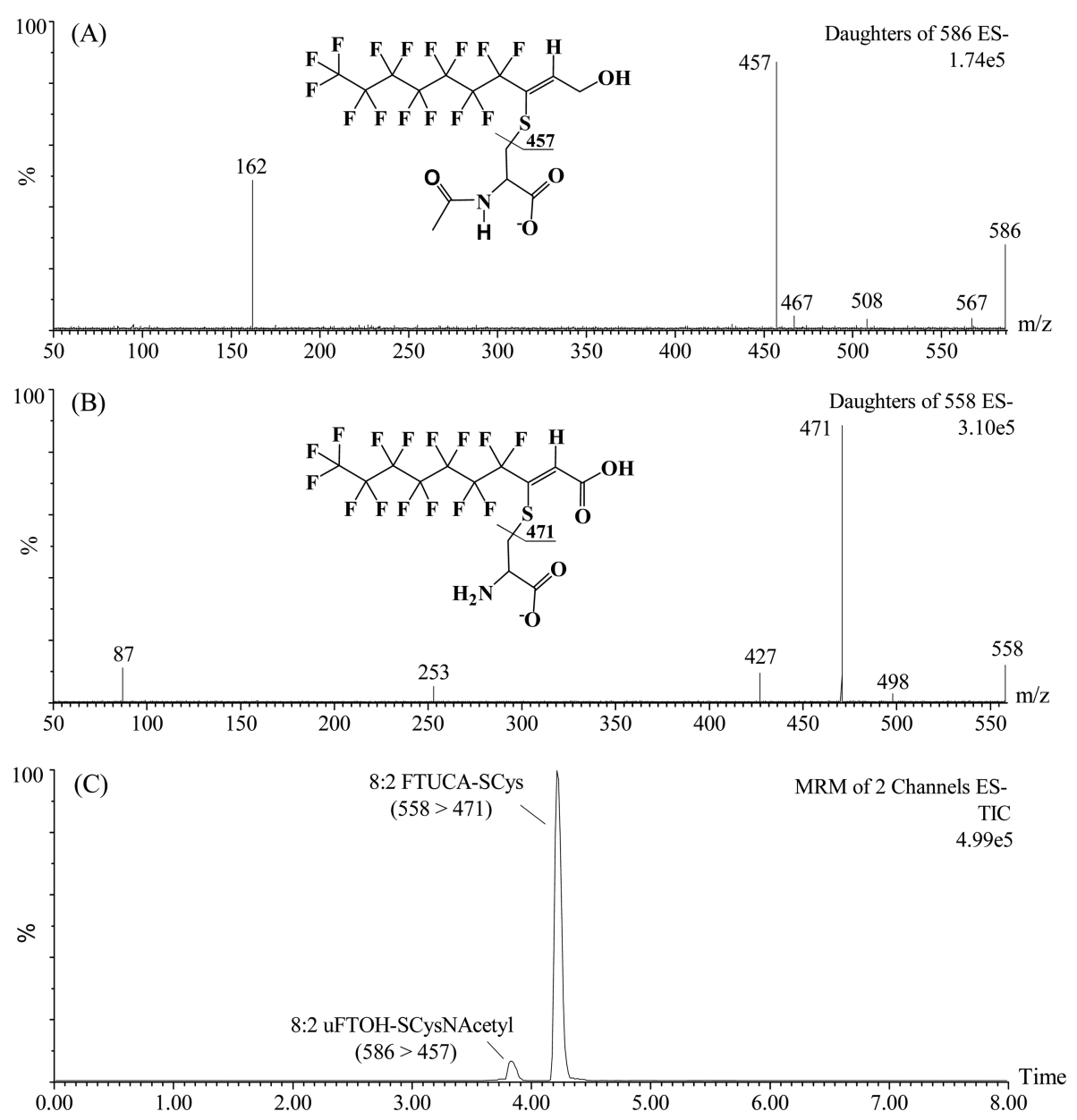

Figure 2. UPLC-MS/MS product ion spectra and chromatograms of GSH-conjugated metabolites of 8:2 FTOH in soybean roots. (A) Product ion spectra of the $m / z 586$ peak (3.82 min), the product was identified as uFTOH-SCysNAcetyl. (B) Product ion spectra of the $m / z 558$ peak (4.22 min), the product was identified as 8:2 FTUCA-SCys. (C) Chromatograms of 8:2 uFTOH-SCysNAcetyl and 8:2 FTUCA-SCys in soybean roots in MRM mode.

Root exudates generally contain enzymes, amino acids, organic acids, and many other chemicals. It was reported that 8:2 FTOH could not support bacterial growth, so that microbial transformation of 8:2 FTOH occurred via cometabolic processes. ${ }^{12}$ Lewis et al. ${ }^{39}$ found that addition of a small dose of carbon source increased the microbial degradation rate. The root exudates can act as carbon sources, which accelerate microbial growth and potentially promote microbial degradation of organic pollutants. ${ }^{40}$ Some of these chemicals were identified as microbial enzyme inducers. The inducers affected the extent of FTOH microbial degradation and the choice of microbial degradation pathways. ${ }^{41,42}$ Concentrations of 8:2 FTOH and total contents of metabolites were 1.18 and $69.2 \mathrm{nmol} \cdot \mathrm{L}^{-1}$, respectively, in solutions of root exudate controls after $144 \mathrm{~h}$ exposure (Table 1 ). When $\mathrm{NaN}_{3}$, a microbial inhibitor, was added, the 8:2 FTOH level increased to $32.8 \mathrm{nmol} \cdot \mathrm{L}^{-1}$, while the total content of degradation products decreased to $13.9 \mathrm{nmol} \cdot \mathrm{L}^{-1}$. No degradation product was found in autoclaved treatments. These results strongly suggested that 8:2 FTOH in solutions was degraded by microbes, whose activities were enhanced by root exudates. In root exudate controls, 7:3 FTCA (24.0 nmol. $\left.\mathrm{L}^{-1}\right)$ and 7:2 sFTOH $\left(23.6 \mathrm{nmol} \cdot \mathrm{L}^{-1}\right)$ were the most abundant metabolites, while PFHpA and PFOA were their downstream metabolites, respectively. ${ }^{4}$ The content of PFHpA was 2.4 times higher than that of PFOA at $144 \mathrm{~h}$ in the root exudate controls. This phenomenon differed from that of whole soybean systems, in which the PFOA level in solutions was 7.6 times as high as that of PFHpA. These differences suggested that inoculation of soybean increased the production of PFOA in solution, and transformation of 7:3 FTCA to PFHpA was mainly ascribed to 
microbial metabolism in solution. It should be pointed out that the influence of microbial degradation of 8:2 FTOH in root area was not easy to be separated from the degradation by plants. Plant root adsorption or absorption of microbial degradation products may lead to the overestimation of the types and amounts of metabolites in plants. In whole soybean systems, trace amounts of PFHpA and PFHxA were found in solutions and soybean roots, but not in stems and leaves (SI Table S10). Moreover, PFHpA and PFHxA levels in root exudate controls were 1.1-11.6 times those in whole soybean systems. These suggested that PFHpA and PFHxA detected in plant roots mainly came from adsorption or absorption from the culture solutions.

GSH-Conjugated Metabolites in Soybean Tissues. GSH-conjugated metabolites in soybean were detected by UPLC-MS/MS based on the studies by Martin et al. ${ }^{21}$ and Fasano et al. ${ }^{15,43}$ The structures of the $8: 2 \mathrm{uFTOH}$ N-acetyl cysteine conjugate (8:2 uFTOH-SCysNAcetyl) and 8:2 FTUCA cysteine conjugate (8:2 FTUCA-SCys) were elucidated (Figure $2)$. Other GSH-conjugated metabolites were not identified. These two conjugated metabolites have been observed during the metabolism of $8: 2 \mathrm{FTOH}$ in rats, which formed through the metabolism of GSH conjugates, 8:2 FTUAL-GSH and 8:2 FTUCA-GSH, respectively. ${ }^{15,43}$ Time-dependent conjugated metabolite levels in plant tissues were monitored based on their peak areas. 8:2 uFTOH-SCysNAcetyl reached peak level at $72 \mathrm{~h}$ (root, stem B and leaf) or $96 \mathrm{~h}$ (stem A), while 8:2 FTUCASCys in all tissues kept increasing during the exposure time (SI Figure S4). Detection of these two GSH-conjugated metabolites suggested that phase II conjugation reactions occurred in soybean during 8:2 FTOH exposure.

Degradation Kinetics and Mass Balance. 8:2 FTOH levels in unplanted controls, root exudate controls, and whole soybean systems decreased with increasing exposure time (Figure 3). Decreases of 8:2 FTOH in the solutions of unplanted

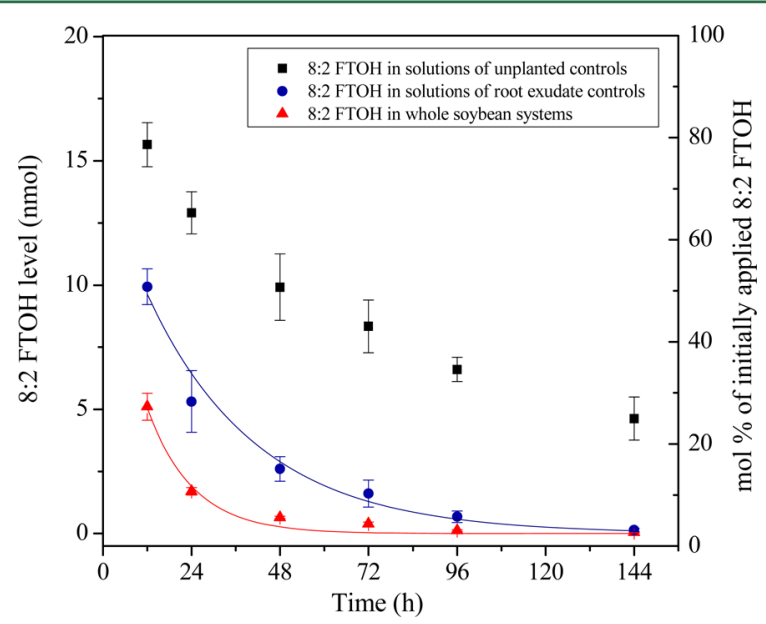

Figure 3. Time-dependent levels of 8:2 FTOH in different exposure experiments. 8:2 FTOH in whole soybean systems includes the levels in each part of soybean plants and in the solution.

controls were due to evaporation within the reactors. The levels of $8: 2 \mathrm{FTOH}$ in whole soybean systems were the sum of the $8: 2$ FTOH amounts in solutions and in plants. The decreases of 8:2 FTOH in root exudate controls were the result of volatilization and microbial degradation, whereas the decreases in whole soybean systems were the result of volatilization, microbial and plant degradation. A first-order kinetic model was applied to assess the decrease of 8:2 FTOH in whole soybean systems and root exudate controls:

$$
q_{\mathrm{t}}=q_{\mathrm{m}} \exp (-k t)
$$

where $q_{\mathrm{m}}$ and $q_{\mathrm{t}}(\mathrm{nmol})$ are the maximum and the level of $8: 2$ FTOH over the exposure time $t(\mathrm{~h})$, and $k\left(\mathrm{~h}^{-1}\right)$ is the transformation rate constant of 8:2 FTOH. The kinetics of the decrease of 8:2 FTOH fit the first-order kinetic model well (eq $1)$, with $\mathrm{R}^{2}$ values higher than $0.978(P<0.001$, Figure 3, SI Table $S 11)$. The calculated $k$ value of whole soybean systems was 2.5 times that of root exudate controls, showing the fast degradation rate of 8:2 FTOH in the presence of plants.

The mass balance of whole soybean systems was tested at each time point by calculating the percentage of total mass of $8: 2$ FTOH and metabolites in the solution and soybean to the initial amount of 8:2 FTOH applied. The recoveries ranged from 15.0 mol \% to $46.3 \mathrm{~mol} \%$ for whole soybean systems. Similar low mass balance was reported for microbial degradation of 8:2 $\mathrm{FTOH}^{44}$ which may have been the result of the inability to quantify some known metabolites, the production of unidentified metabolites, and the potential production of nonextractable metabolites that were covalently bound to biological macromolecules. ${ }^{5}$ The recoveries of whole soybean systems were much lower than those of unplanted controls $(23.1-78.1 \mathrm{~mol} \%)$ and root exudate controls (42.1-67.3 mol \%), and decreased with increasing exposure time (SI Table S10), suggesting that the formation of unquantifiable, unknown, and/or unrecovered bound metabolites resulted from soybean metabolism. Some intermediate or stable products reported elsewhere, such as 8:2 FTAL $\left[\mathrm{F}\left(\mathrm{CF}_{2}\right)_{8} \mathrm{CH}_{2} \mathrm{CHO}\right], 7: 3$ FTUCA $\left[\mathrm{F}\left(\mathrm{CF}_{2}\right)_{7} \mathrm{CH}=\right.$ $\mathrm{CHCOOH}]$, and 2H-PFOA $\left[\mathrm{F}\left(\mathrm{CF}_{2}\right)_{6} \mathrm{CFHCOOH}\right]$, ${ }^{13}$ were not monitored in the present work due to the standards being unavailable in our laboratory. The increasing trends of conjugated metabolite levels in soybean (SI Figure S4) demonstrated that the formation of conjugated metabolites, at least partially, was responsible for the decreases in recovery.

Enzyme Responses. ADH, ALDH, GST, and CYP450 are enzymes that take part in metabolizing organic pollutants in plants. ${ }^{45-47}$ In order to explore the roles of these enzymes in metabolizing 8:2 FTOH in soybean, dynamic changes of $\mathrm{ADH}$, ALDH, GST, and CYP450 activities in soybean roots during the exposure time were investigated, because roots were the tissues with the most abundant metabolites (Figure 1A, SI Table S10). Activities of $\mathrm{ADH}, \mathrm{ALDH}$, and GST in soybean roots increased over the exposure time until 48,72 , and $72 \mathrm{~h}$, respectively, and then decreased (Figure 4A-C). Compared with those of blank controls, $\mathrm{ADH}, \mathrm{ALDH}$, and GST activities at each time point significantly increased $(P<0.05)$, which were $1.27-1.80,1.17-$ 1.61 , and 1.19-1.68 times as high as those of blank controls, respectively. The obvious increases of $\mathrm{ADH}, \mathrm{ALDH}$, and GST activities demonstrated that these enzymes were involved in the metabolism of 8:2 FTOH in soybean. ADH and ALDH most likely sequentially catalyzed the phase I oxidation of $8: 2 \mathrm{FTOH}$ to $8: 2$ FTAL, and then to $8: 2$ FTCA. $^{15}$ GST was reported to play a role in 8:2 FTOH phase II metabolism through the formation of glutathione conjugates. ${ }^{15-17}$ The increases in GST activities were consistent with the finding of GSH-conjugated metabolites in soybean.

The CYP450 superfamily includes a large group of enzymes that have been found in all domains of life. They are thought to catalyze the major phase I oxidation reactions of many xenobiotics. $^{47}$ In vitro studies have suggested that CYP450 enzymes participate in the oxidation of FTOHs by activating 

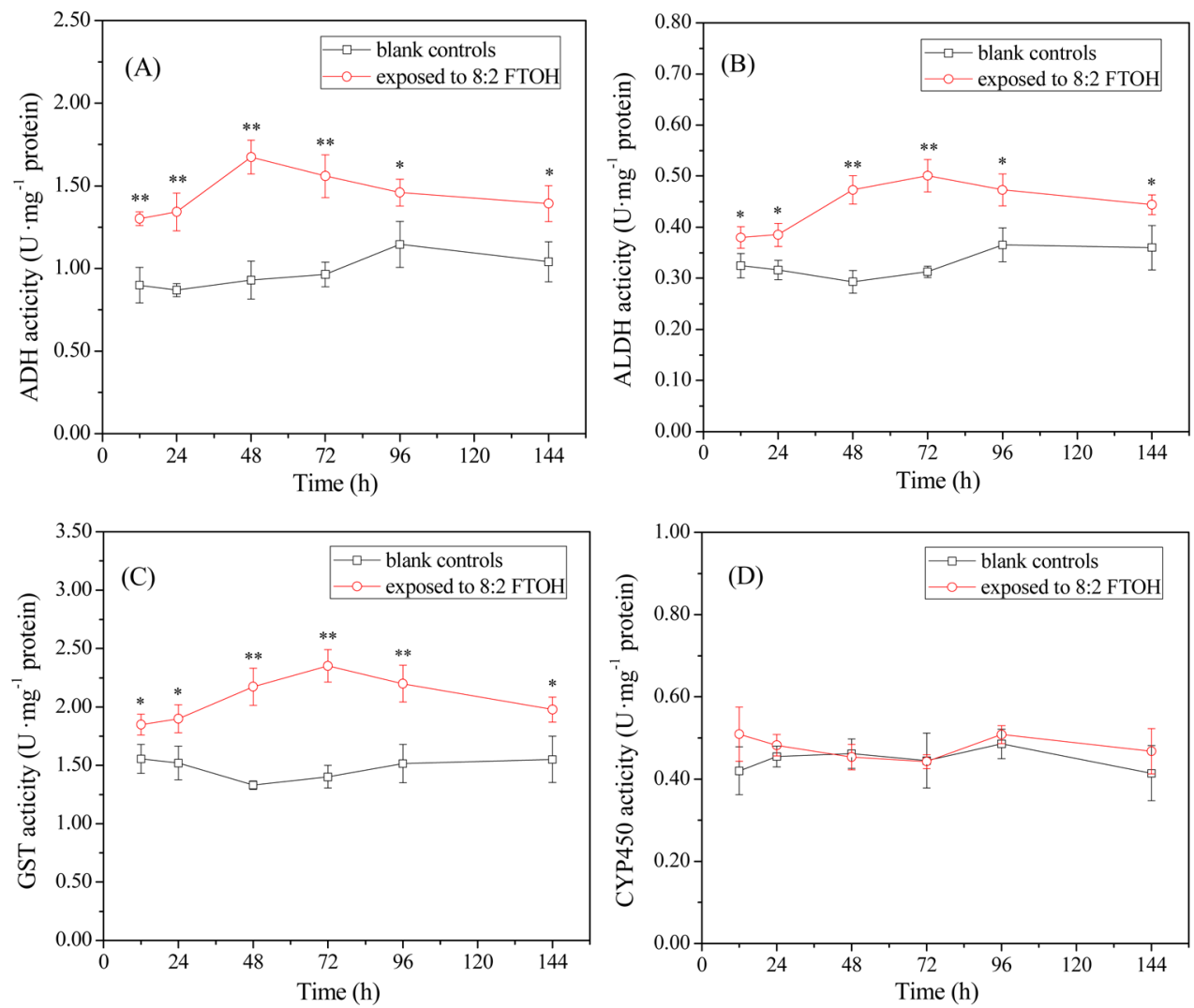

Figure 4. Dynamic changes of ADH (A), ALDH (B), GST (C), and CYP450 (D) activities in soybean roots. Statistics were calculated with a two-tailed unpaired Student's $t$ test. Statistical significance relative to blank controls at the same time point is indicated by $* P<0.05$, ** $P<0.01$.

molecular oxygen. ${ }^{20,48}$ Martin et al. ${ }^{48}$ reported that CYP2E1, one subfamily of CYP450, likely catalyzed the initial oxidation of FTOH to the respective aldehyde in isolated rat hepatocytes. $\mathrm{Li}$ et al. ${ }^{20}$ found that among 11 isoforms of human CYP450 studied, only CYP2C19 was capable of catalyzing 8:2 FTOH phase I metabolism. However, in this study, 8:2 FTOH treatment did not change the CYP450 activity in soybean roots significantly $(P$ $>0.05$, Figure 4D), which indicated that CYP450 may not be the key enzyme involved in 8:2 FTOH metabolism in soybean. It should also be pointed out that lack of change in total CYP450 activity may not reflect the role of the subfamily involved. The activities of some isoenzymes are induced, while others may be inhibited during xenobiotic metabolism. ${ }^{49}$ The role of CYP450 in 8:2 FTOH metabolism by plants thus requires further investigation.

Metabolic Pathways of 8:2 FTOH in Soybean. Much attention has been paid to the metabolism of FTOHs in different biological systems. ${ }^{4,5,19}$ However, limited information is available on the metabolic pathways in plants. In this study, phase I and phase II metabolism of $8: 2 \mathrm{FTOH}$ in soybean were revealed. The finding of 8:2 FTCA, 8:2 FTUCA, 7:2 sFTOH, PFOA, and 7:3 FTCA in all tissues of soybean, and the increases of ADH, ALDH enzyme activities, suggested that phase I metabolic pathways of 8:2 FTOH in soybean were similar to those of microbes and animals. ${ }^{13,15,17}$ PFNA has been reported to be one of the stable products obtained via $\alpha$-oxidation of 8:2 FTCA in animal-based biotransformation studies. ${ }^{15-18}$ However, PFNA was not observed in soybean tissues, which suggested that $\alpha$-oxidation of 8:2 FTCA in soybean did not occur. This result was consistent with that obtained by Rakin et al., ${ }^{28}$ who investigated the biodegradability of a fluorotelomer-based acrylate polymer in a soil-plant microcosm. 7:3 FTCA was considered to be a terminal metabolite in soil systems. ${ }^{13}$ The dealkylation and defluorination steps of 7:3 FTCA could yield low levels of PFHpA. ${ }^{16,17,50}$ PFHxA was observed in aerobic microbial degradation of $8: 2 \mathrm{FTOH}$ through the removal of two perfluorocarbons from 7:3 FTUCA. ${ }^{12,13,51}$ In this study, PFHpA and PFHxA in soybean roots were proved to be resulted from adsorption or absorption from the culture solutions. It has been reported that conjugation is the major metabolic pathway of 8:2 FTOH in human liver microsomes. ${ }^{20}$ The increases of GSHconjugated metabolite levels and GST enzyme activities in soybean tissues during the exposure time suggested the importance of conjugation on 8:2 FTOH metabolism. To our knowledge, this is the first study to monitor the conjugated metabolites of $8: 2 \mathrm{FTOH}$ in a plant. Based on the above analysis, metabolic pathways of 8:2 FTOH in soybean were proposed (SI Figure S5). PFOA was the only terminal PFCA product in soybean, which may be in part due to the relatively short exposure time of $144 \mathrm{~h}$. Long-term experiment is limited by the relatively small volume of the exposure reactor $(150 \mathrm{~mL})$. It should be noted that the formation of PFCAs from biodegradation of 8:2 FTOH needs multiple steps. ${ }^{13,15,17}$ Prolonging exposure time may lead to different composition patterns of the final degradation products.

Environmental Implications. This study provides, for the first time, direct evidence for the uptake, accumulation and metabolism of 8:2 FTOH in plants. Uptake and translocation of 8:2 FTOH from roots to stems and leaves indicates its potential transportation and accumulation in the food chain. Transformation of 8:2 FTOH to per- and poly fluorinated metabolites in plants may have implications for toxicity assessment, 
depending on the nature of the metabolites and their potential to cause adverse effects. The information presented here is useful to improve current understanding of the fates of FTOHs and fluorotelomer-based compounds, as well as the source of PFCAs in the environment. Future research will include exploration of the detailed enzymatic pathways leading to the removal of multiple $-\mathrm{CF}_{2}-$ groups, as well as potential transpiration of volatile and semivolatile FTOHs.

\section{ASSOCIATED CONTENT}

\section{S Supporting Information}

The Supporting Information is available free of charge on the ACS Publications website at DOI: 10.1021/acs.est.6b03734.

Additional information as noted in the text (PDF)

\section{AUTHOR INFORMATION}

\section{Corresponding Author}

*Phone: +86-10-62849329; fax: +86-10-62923563; e-mail: bwen@rcees.ac.cn.

\section{ORCID}

Bei Wen: 0000-0002-6380-0123

\section{Notes}

The authors declare no competing financial interest.

\section{ACKNOWLEDGMENTS}

We thank the National Natural Science Foundation of China (Projects 21537005, 41371460, 41671465, and 21321004), and the Strategic Priority Research Program of the Chinese Academy of Sciences (Grant XDB14020202) for financial support.

\section{REFERENCES}

(1) Lindstrom, A. B.; Strynar, M. J.; Libelo, E. L. Polyfluorinated compounds: Past, present, and future. Environ. Sci. Technol. 2011, 45 (19), 7954-7961.

(2) Prevedouros, K.; Cousins, I. T.; Buck, R. C.; Korzeniowski, S. H. Sources, fate and transport of perfluorocarboxylates. Environ. Sci. Technol. 2006, 40 (1), 32-44.

(3) Fromel, T.; Knepper, T. P. Biodegradation of fluorinated alkyl substances. Rev. Environ. Contam. Toxicol. 2010, 208, 161-77.

(4) Liu, J. X.; Avendano, S. M. Microbial degradation of polyfluoroalkyl chemicals in the environment: A review. Environ. Int. 2013, 61, 98-114.

(5) Butt, C. M.; Muir, D. C. G.; Mabury, S. A. Biotransformation pathways of fluorotelomer-based polyfluoroalkyl substances: A review. Environ. Toxicol. Chem. 2014, 33 (2), 243-267.

(6) Dinglasan-Panlilio, M. J. A.; Mabury, S. A. Significant residual fluorinated alcohols present in various fluorinated materials. Environ. Sci. Technol. 2006, 40 (5), 1447-1453.

(7) Russell, M. H.; Berti, W. R.; Szostek, B.; Buck, R. C. Investigation of the biodegradation potential of a fluoroacrylate polymer product in aerobic soils. Environ. Sci. Technol. 2008, 42 (3), 800-807.

(8) Russell, M. H.; Berti, W. R.; Szostek, B.; Wang, N.; Buck, R. C. Evaluation of PFO formation from the biodegradation of a fluorotelomer-based urethane polymer product in aerobic soils. Polym. Degrad. Stab. 2010, 95 (1), 79-85.

(9) Ellis, D. A.; Martin, J. W.; De Silva, A. O.; Mabury, S. A.; Hurley, M. D.; Andersen, M. P. S.; Wallington, T. J. Degradation of fluorotelomer alcohols: A likely atmospheric source of perfluorinated carboxylic acids. Environ. Sci. Technol. 2004, 38 (12), 3316-3321.

(10) Wallington, T. J.; Hurley, M. D.; Xia, J.; Wuebbles, D. J.; Sillman, S.; Ito, A.; Penner, J. E.; Ellis, D. A.; Martin, J.; Mabury, S. A.; Nielsen, O. J.; Andersen, M. P. S. Formation of $\mathrm{C}_{7} \mathrm{~F}_{15} \mathrm{COOH}$ (PFOA) and other perfluorocarboxylic acids during the atmospheric oxidation of $8: 2$ fluorotelomer alcohol. Environ. Sci. Technol. 2006, 40 (3), 924-930.
(11) Gauthier, S. A.; Mabury, S. A. Aqueous photolysis of 8:2 fluorotelomer alcohol. Environ. Toxicol. Chem. 2005, 24 (8), 18371846.

(12) Liu, J. X.; Lee, L. S.; Nies, L. F.; Nakatsu, C. H.; Turco, R. F. Biotransformation of 8:2 fluorotelomer alcohol in soil and by soil bacteria isolates. Environ. Sci. Technol. 2007, 41 (23), 8024-8030.

(13) Wang, N.; Szostek, B.; Buck, R. C.; Folsom, P. W.; Sulecki, L. M.; Gannon, J. T. 8-2 Fluorotelomer alcohol aerobic soil biodegradation: Pathways, metabolites, and metabolite yields. Chemosphere 2009, 75 (8), 1089-1096.

(14) Zhang, S.; Szostek, B.; McCausland, P. K.; Wolstenholme, B. W.; Lu, X. X.; Wang, N.; Buck, R. C. 6:2 and 8:2 Fluorotelomer alcohol anaerobic biotransformation in digester sludge from a WWTP under methanogenic conditions. Environ. Sci. Technol. 2013, 47 (9), 42274235.

(15) Fasano, W. J.; Carpenter, S. C.; Gannon, S. A.; Snow, T. A.; Stadler, J. C.; Kennedy, G. L.; Buck, R. C.; Korzeniowski, S. H.; Hinderliter, P. M.; Kemper, R. A. Absorption, distribution, metabolism, and elimination of 8-2 fluorotelomer alcohol in the rat. Toxicol. Sci. 2006, 91 (2), 341-355.

(16) Nabb, D. L.; Szostek, B.; Himmelstein, M. W.; Mawn, M. P.; Gargas, M. L.; Sweeney, L. M.; Stadler, J. C.; Buck, R. C.; Fasano, W. J. In vitro metabolism of 8-2 fluorotelomer alcohol: Interspecies comparisons and metabolic pathway refinement. Toxicol. Sci. 2007, 100 (2), 333-344.

(17) Butt, C. M.; Muir, D. C. G.; Mabury, S. A. Elucidating the pathways of poly- and perfluorinated acid formation in rainbow trout. Environ. Sci. Technol. 2010, 44 (13), 4973-4980.

(18) Henderson, W. M.; Smith, M. A. Perfluorooctanoic acid and perfluorononanoic acid in fetal and neonatal mice following in utero exposure to 8-2 fluorotelomer alcohol. Toxicol. Sci. 2007, 95 (2), 452461.

(19) Nilsson, H.; Karrman, A.; Rotander, A.; van Bavel, B.; Lindstrom, G.; Westberg, H. Biotransformation of fluorotelomer compound to perfluorocarboxylates in humans. Environ. Int. 2013, 51, 8-12.

(20) Li, Z. M.; Guo, L. H.; Ren, X. M. Biotransformation of 8:2 fluorotelomer alcohol by recombinant human cytochrome P450s, human liver microsomes and human liver cytosol. Environ. Sci.: Processes Impacts 2016, 18, 538-546.

(21) Martin, J. W.; Mabury, S. A.; O’Brien, P. J. Metabolic products and pathways of fluorotelomer alcohols in isolated rat hepatocytes. Chem.Biol. Interact. 2005, 155 (3), 165-180.

(22) Higgins, C. P.; Field, J. A.; Criddle, C. S.; Luthy, R. G. Quantitative determination of perfluorochemicals in sediments and domestic sludge. Environ. Sci. Technol. 2005, 39 (11), 3946-3956.

(23) Sindiku, O.; Orata, F.; Weber, R.; Osibanjo, O. Per- and polyfluoroalkyl substances in selected sewage sludge in Nigeria. Chemosphere 2013, 92 (3), 329-335.

(24) Sepulvado, J. G.; Blaine, A. C.; Hundal, L. S.; Higgins, C. P. Occurrence and fate of perfluorochemicals in soil following the land application of municipal biosolids. Environ. Sci. Technol. 2011, 45 (19), $8106-8112$

(25) Washington, J. W.; Yoo, H.; Ellington, J. J.; Jenkins, T. M.; Libelo, E. L. Concentrations, distribution, and persistence of perfluoroalkylates in sludge-applied soils near Decatur, Alabama, USA. Environ. Sci. Technol. 2010, 44 (22), 8390-8396.

(26) Yoo, H.; Washington, J. W.; Ellington, J. J.; Jenkins, T. M.; Neill, M. P. Concentrations, distribution, and persistence of fluorotelomer alcohols in sludge-applied soils near Decatur, Alabama, USA. Environ. Sci. Technol. 2010, 44 (22), 8397-8402.

(27) Zhang, H. N.; Wen, B.; Hu, X. Y.; Wu, Y. L.; Luo, L.; Chen, Z. E.; Zhang, S. Z. Determination of fluorotelomer alcohols and their degradation products in biosolids-amended soils and plants using ultra-high performance liquid chromatography tandem mass spectrometry. J. Chromatogr. A 2015, 1404, 72-80.

(28) Rankin, K.; Lee, H.; Tseng, P. J.; Mabury, S. A. Investigating the biodegradability of a fluorotelomer-based acrylate polymer in a soilplant microcosm by indirect and direct analysis. Environ. Sci. Technol. 2014, 48 (21), 12783-12790. 
(29) Hoagland, D. R.; Arnon, D. I. The water-culture method for growing plants without soil. Calif. Agric. Exp. Sta. Circ. 1950, 347, 1-32. (30) Bradford, M. M. A rapid and sensitive method for the quantitation of microgram quantities of protein utilizing the principle of protein-dye binding. Anal. Biochem. 1976, 72 (1), 248-254.

(31) Liu, J. X.; Lee, L. S. Solubility and sorption by soils of $8: 2$ fluorotelomer alcohol in water and cosolvent systems. Environ. Sci. Technol. 2005, 39 (19), 7535-7540.

(32) Carmosini, N.; Lee, L. S. Partitioning of fluorotelomer alcohols to octanol and different sources of dissolved organic carbon. Environ. Sci. Technol. 2008, 42 (17), 6559-6565.

(33) Kaiser, M. A.; Cobranchi, D. P.; Kao, C. P. C.; Krusic, P. J.; Marchione, A. A.; Buck, R. C. Physicochemical properties of 8-2 fluorinated Telomer B alcohol. J. Chem. Eng. Data 2004, 49 (4), 912916.

(34) Lei, Y. D.; Wania, F.; Mathers, D.; Mabury, S. A. Determination of vapor pressures, octanol-air, and water-air partition coefficients for polyfluorinated sulfonamide, sulfonamidoethanols, and telomer alcohols. J. Chem. Eng. Data 2004, 49 (4), 1013-1022.

(35) Wang, N.; Szostek, B.; Folsom, P. W.; Sulecki, L. M.; Capka, V.; Buck, R. C.; Berti, W. R.; Gannon, J. T. Aerobic biotransformation of ${ }^{14} \mathrm{C}$-labeled $8-2$ telomer $\mathrm{B}$ alcohol by activated sludge from a domestic sewage treatment plant. Environ. Sci. Technol. 2005, 39 (2), 531-538.

(36) Collins, C.; Fryer, M.; Grosso, A. Plant uptake of non-ionic organic chemicals. Environ. Sci. Technol. 2006, 40 (1), 45-52.

(37) Li, H.; Sheng, G.; Sheng, W.; Xu, O. Uptake of trifluralin and lindane from water by ryegrass. Chemosphere 2002, 48 (3), 335-341.

(38) Pangesti, N.; Pineda, A.; Pieterse, C. M. J.; Dicke, M.; van Loon, J. J. A. Two-way plant-mediated interactions between root-associated microbes and insects: from ecology to mechanisms. Front. Plant Sci. 2013, 4, DOI 10.3389/fpls.2013.00414.

(39) Lewis, M.; Kim, M. H.; Wang, N.; Chu, K. H. Engineering artificial communities for enhanced FTOH degradation. Sci. Total Environ., 2016, in press; DOI 57293510.1016/j.scitotenv.2016.07.223.

(40) Meng, L.; Zhu, Y. G. Pyrene biodegradation in an industrial soil exposed to simulated rhizodeposition: How does it affect functional microbial abundance? Environ. Sci. Technol. 2011, 45 (4), 1579-1585.

(41) Kim, M. H.; Wang, N.; McDonald, T.; Chu, K. H. Biodefluorination and biotransformation of fluorotelomer alcohols by two alkane-degrading Pseudomonas strains. Biotechnol. Bioeng. 2012, 109 (12), 3041-3048.

(42) Kim, M. H.; Wang, N.; Chu, K. H. 6:2 Fluorotelomer alcohol (6:2 FTOH) biodegradation by multiple microbial species under different physiological conditions. Appl. Microbiol. Biotechnol. 2014, 98 (4), $1831-1840$

(43) Fasano, W. J.; Sweeney, L. M.; Mawn, M. P.; Nabb, D. L.; Szostek, B.; Buck, R. C.; Gargas, M. L. Kinetics of 8-2 fluorotelomer alcohol and its metabolites, and liver glutathione status following daily oral dosing for 45 days in male and female rats. Chem.-Biol. Interact. 2009, 180 (2), 281-295.

(44) Dinglasan, M. J. A.; Ye, Y.; Edwards, E. A.; Mabury, S. A. Fluorotelomer alcohol biodegradation yields poly- and perfluorinated acids. Environ. Sci. Technol. 2004, 38 (10), 2857-2864.

(45) Mena-Benitez, G. L.; Gandia-Herrero, F.; Graham, S.; Larson, T. R.; McQueen-Mason, S. J.; French, C. E.; Rylott, E. L.; Bruce, N. C. Engineering a catabolic pathway in plants for the degradation of 1,2dichloroethane. Plant Physiol. 2008, 147 (3), 1192-1198.

(46) Huang, H. L.; Zhang, S. Z.; Wang, S.; Lv, J. T. In vitro biotransformation of PBDEs by root crude enzyme extracts: Potential role of nitrate reductase $(\mathrm{NaR})$ and glutathione S-transferase (GST) in their debromination. Chemosphere 2013, 90 (6), 1885-1892.

(47) Zhai, G.; Lehmler, H. J.; Schnoor, J. L. Inhibition of cytochromes $\mathrm{P} 450$ and the hydroxylation of 4-monochlorobiphenyl in whole poplar. Environ. Sci. Technol. 2013, 47 (13), 6829-6835.

(48) Martin, J. W.; Chan, K.; Mabury, S. A.; O’Brien, P. J. Bioactivation of fluorotelomer alcohols in isolated rat hepatocytes. Chem.-Biol. Interact. 2009, 177 (3), 196-203.
(49) Siess, M. H.; Le Bon, A. M.; Canivenc-Lavier, M. C.; Suschetet, M. Modification of hepatic drug-metabolizing enzymes in rats treated with alkyl sulfides. Cancer Lett. 1997, 120 (2), 195-201.

(50) Wang, N.; Buck, R. C.; Szostek, B.; Sulecki, L. M.; Wolstenholme, B. W. 5:3 Polyfluorinated acid aerobic biotransformation in activated sludge via novel "one-carbon removal pathways. Chemosphere 2012, 87 (5), 527-534.

(51) Wang, N.; Szostek, B.; Buck, R. C.; Folsom, P. W.; Sulecki, L. M.; Capka, V.; Berti, W. R.; Gannon, J. T. Fluorotelomer alcohol biodegradation - Direct evidence that perfluorinated carbon chains breakdown. Environ. Sci. Technol. 2005, 39 (19), 7516-7528. 